\title{
A STUDY OF TIME AND SPACE CORRELATIONS IN FIELD EMISSION CURRENT FLUCTUATIONS WITH A FIBER-OPTICAL TECHNIQUE
}

\author{
A.A. Dadykin and A.G. Naumovets \\ Institute of Physics, Ukrainian Academy of Sciences \\ Prospekt Nauki 46, 252650 Kiev 28, USSR \\ (Received May 21, 1991; in final version July 19, 1991)
}

\begin{abstract}
A new technique of measurements of field emission current fluctuations is proposed. The technique uses fiber optics and offers such advantages as the possibility to easily vary the geometry of the probed regions and the distance between them on the field emitter. The correlation functions of field emission current fluctuations have been measured with this technique for $\mathrm{O} / \mathrm{W}(110)$, $\mathrm{H} / \mathrm{W}(110), \mathrm{Si}(111)$ and $\mathrm{Na} / \mathrm{Si}(111)$ systems. Some data on surface diffusion coefficients are derived from the measurements.
\end{abstract}

PACS numbers: $79.70 .+q, 05.40 .+j$

\section{Introduction}

The idea about utilization of fluctuation measurements to gain knowledge about the kinetics of irreversible processes stems from the works of M. Smoluchowski. It was extended by L. Onsager who put forward a hypothesis that long-wave microscopic density fluctuations of an equilibrium state decay in compliance with macroscopic laws. Ch. Kleint, R. Gomer, R. Męclewski, L. Swanson and their coworkers developed efficient methods for the investigation of surface diffusion kinetics based on the measurements of field emission current fluctuations (FECF) (see e.g. Refs. [1-7]). Information about surface kinetics can be extracted from spectral density and correlation functions of FECF, in particular from the time (auto)correlation function

$$
R_{x}(\tau)=\lim _{T \rightarrow \infty} \frac{1}{T} \int_{0}^{T} \delta i_{x}(t) \delta i_{x}(t+\tau) \mathrm{d} t,
$$

the cross-correlation function

$$
R_{x y}(\tau)=\lim _{T \rightarrow \infty} \frac{1}{T} \int_{0}^{T} \delta i_{x}(t) \delta i_{y}(t+\tau) \mathrm{d} t
$$

and the space correlation function

$$
R(\rho)=\lim _{L \rightarrow \infty, t=\text { const. }} \frac{1}{L} \int_{0}^{L} \delta i(r) \delta i(r+\rho) \mathrm{d} r .
$$


Here $x$ and $y$ label the regions on the surface probed in the measurements, $\delta i$ is the current fluctuation, $t$ - the time, $\tau$ - the time interval between current measurements, $T$ - the total sampling time, $r$ - the coordinate, $\rho$ - the distance between the probed regions, and $L$ - the size of the averaging region.

The devices used so far for FECF measurements were E.W. Müller's field emission microscopes provided with one or two probe holes in the screen through which the electrons from the probed area(s) on the tip are directed to the current collector(s). Field emission microscopes with one probe hole are utilized when the spectral density function or autocorrelation function (1) are determined $[1,2]$. In the measurements of the cross-correlation function (2) the tubes with two probe holes are employed [8-13].

In order to extend the potentialities of the FECF method it is desirable to construct a field emission tube in which some important additional measurements can be made.

First, it would be profitable to have a possibility to vary the size of the region probed on the tip surface. This is important in order to make the interpretation of the autocorrelation functions more conclusive (see Sec. 3). Anyway, it must be kept in mind that in this interpretation an inverse problem is considered.

Second, in the study of cross-correlation it is important to measure the dependence of the cross-correlation coefficient on the spacing between the probed regions. In the original version of the field emission tube in which the first measurements of FECF cross-correlations were carried out the distance between the probe holes was fixed [8]. Recently a tube was constructed in which this distance can be changed continuously. Nevertheless the range of this variation is somewhat limited [10, 12].

Third, an inherent feature of the FECF method is the presence of a rather high electric field $\left(\sim 10^{7} \mathrm{~V} / \mathrm{cm}\right)$ at the surface under study. In principle this can affect the kinetics of surface processes manifesting themselves in the current fluctuations, particularly in the case when adparticles possess an appreciable dipole moment and/or polarizability. Obviously, this effect can not be avoided completely, but it is desirable to minimize the field and emission current e.g. by passing to an electron counting mode.

In this work we report on a new version of the FECF method which utilizes fiber optics and ensures the possibilities listed above.

\section{Experimental}

\subsection{Apparatus}

A scheme of our experimental tube is depicted in Fig. 1. This is an all-glass field emission microscope pumped down to a base pressure of $\sim 10^{-10}$ Torr. The tip can be cleaned by passing a current through the heating loop and/or by field evaporation. It can also be cooled with liquid nitrogen. The field emitted electrons pass through an electrostatic lens serving to control the size of the pattern on the screen and enter a microchannel plate. The application of the microchannel plate allows to carry out the fluctuation measurements in the electron counting 

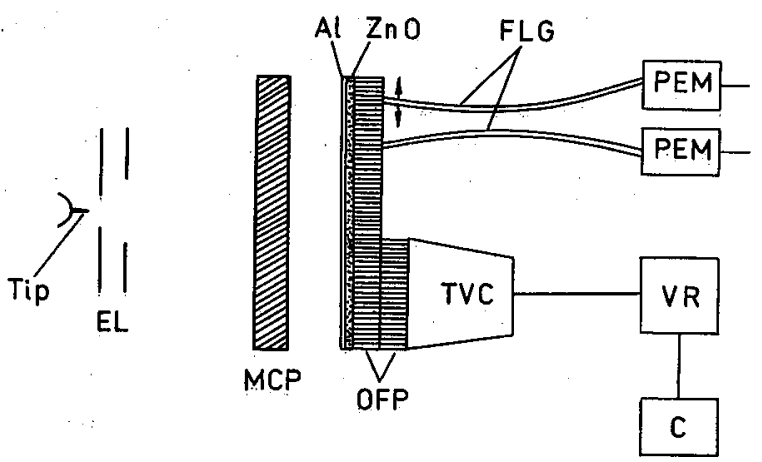

Fig. 1. Schematic diagram of experimental arrangement. EL is an electrostatic lens, MCP - a microchannel plate, OFP - optical-fiber plates, FLG - flexible fiber light guides, PEM - photoelectric multipliers, TVC - a videocamera with optical-fiber entrance window, VR - a videorecorder and $\mathrm{C}-$ an X6-4 correlator. The screen is made of $\mathrm{ZnO}$ phosphor covered with an Al film.

mode thereby reducing the current by 3 to 4 orders of magnitude as compared to usual collector measurements (of course electron multiplication can also be used in conventional FE tubes with probe holes - see e.g. Ref. [14]). The electrons leaving the microchannel plate are accelerated to $\approx 10 \mathrm{keV}$ and they impinge on a $\mathrm{ZnO}$ phosphor screen covered with an electron-transparent aluminium film $(\approx 1500 \AA$ thick $)$. This film serves to apply a potential to the screen to suppress secondary electron emission from the phosphor and to enhance the light output of the screen which was found to be linear with the current within 2 to 3 orders of its magnitude.

The phosphor is evaporated immediately on the face of the tube which is a vacuum-tight optic-fiber plate. In the measurements of the autocorrelation and cross-correlation functions the light signal is picked up from the outer face of the screen with one or two flexible optic-fiber waveguides and conveyed to the photomultipliers. (The combination phosphor + phototube is also used in the FECF measurements made with probe-hole field emission tubes - see e.g. Ref. [15].) The size of the region probed on the tip surface can easily be varied by using different entrance diaphragms in the light guides. In order to investigate diffusion anisotropy, Bowman et al. [16] suggested to use a probe hole in the form of a long and narrow rectangular slit. Two sequential fluctuation measurements are made with the slit oriented along the principal axes of the diffusion tensor. Obviously, such experiments can readily be implemented with a fiber guide which has a slit entrance diaphragm. Using two fiber guides, it is easy to determine the cross-correlation function (2) for various spacings between the probed spots.

In order to evaluate the space correlation function (3), one can analyze a field emission pattern recorded with a video camera which can also have an optical-fiber entrance window. The type of the camera tube (vidicon, supervidicon, dissector etc.) is chosen from considerations of sensitivity and recording speed. A conven- 
tional video apparatus is used to form and record a frame. The space correlation function is derived by processing a line within the frame.

The spatial resolution of the system field emission cathode + microchannel plate + screen + fiber window is determined mainly by the resolution of the field emission nicroscope $(\approx 20-30 \AA)$. The time resolution is limited by the $\mathrm{ZnO}$ afterglow $\left(\sim 10^{-6} \mathrm{~s}\right)$.

\subsection{Signal processing}

Figure 2 shows a schematic diagram of a processing channel designed for FECF measurements and extraction of the correlation functions.

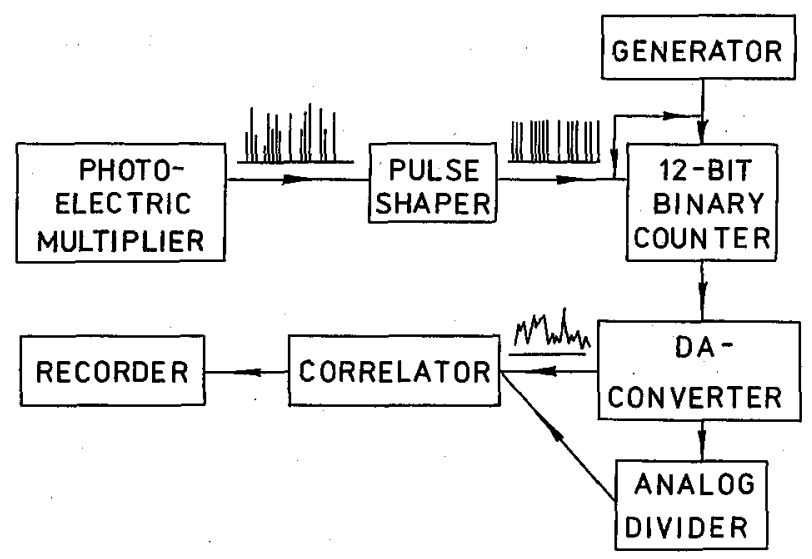

Fig. 2. Schematic diagram of a processing channel.

Signals from the photoelectric multiplier which have the form of separate voltage pulses enter, after amplification and amplitude discrimination, into a pulse shaper. It generates rectangular pulses of standarized height and length and varying repetition frequency. The duration and average repetition frequency of the pulses (the latter being determined by the extracted emission current) are chosen in such a way that a preset measurement accuracy can be ensured within the frequency range of $0.1 \mathrm{IIz}-250 \mathrm{kHz}$. With the aid of a 12 bit binary counter and a digital-to-analog converter the pulse signal is transformed to an analogous one which reproduces the fluctuations of the emission current. Such a conversion is needed, since a digital X6-4 correlator operating in a concurrent mode is used to analyze the signal. The correlator calculates simultaneously 100 points of the correlation function. Typically a few minutes suffice to obtain the correlation function with an accuracy of $5 \%$ under the conditions of our experiments.

In order to ensure a required accuracy of the correlation function measurement within the entire time delay interval, two modes of pulse-to-analog conversion are provided: frequency-to-voltage and pulse interval-to-voltage.

In the first case the counter counts the current pulses within a constant interval $T$ defined by a precision timemark generator. In the second case the pulses 
from the generator are counted which are contained within fluctuating time intervals between the arriving field emission current pulses. In this operation mode, before the correlation analysis, the analog signal obtained from the D-A-converter is transformed by a $f=1 / T$ algorithm with an analog divider.

The apparatus allows to measure the field emission current fluctuations within the frequency band of $0.1 \mathrm{Izz}$ to $10 \mathrm{kIIz}$ at an average current from the probed area of $\sim 10^{-14} \mathrm{~A}$. If the band is to be extended up to $250 \mathrm{kHz}$, the repetition frequency-to-voltage conversion is performed by a capacitor frequency meter with a tunable low-pass filter.

The adjustment of the apparatus (the choice of the multiplication coefficients of the microchannel plate and photoelectric multiplier, of the discrimination level of the pulse shaper, of the time intervals in pulse counting etc.) was implemented by applying test voltage pulses with a known correlation function to the tip.

As first demonstrated by Okano et al. [17], the digital processing of the field emission current fluctuations provides a much better sensitivity and stability in comparison with conventional analog measurements and processing.

\section{Results and discussion}

Below we present the FECF correlation functions for various systems obtained with the fiber-optics technique (FOT). For testing purposes FOT was applied to some systems which were studied in detail earlier with conventional technique using tubes with probe holes and current collectors.

\subsection{Oxygen on $W(110)$}

Figure 3 slows the FECF autocorrelation functions measured for an $\mathrm{O} / \mathrm{W}(110)$ system. The data were obtained at $T=600 \mathrm{~K}$ and a degree of coverage

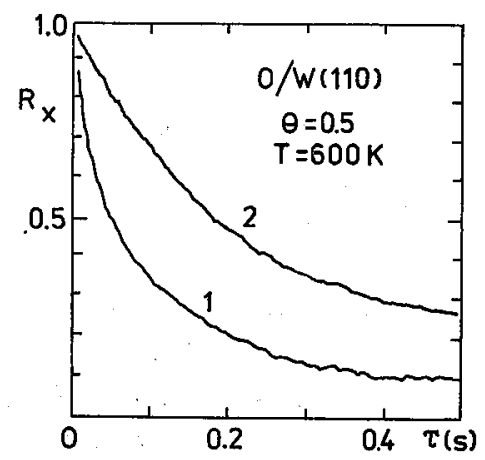

Fig. 3. Autocorrelation functions (in arbitrary units) of field emission current fluctuations for the $\mathrm{O} / \mathrm{W}(110)$ system. Probed area radii are $50 \AA(1)$ and $100 \AA$ (2). Electron counting rates are $9 \times 10^{4} \mathrm{~s}^{-1}(1)$ and $1 \times 10^{5} \mathrm{~s}^{-1}(2)$.

of $\Theta=0.5$ for two probed regions with the radii $r_{01}=50 \AA$ and $r_{02}=100 \AA$. 
It should be recalled that the FECF autocorrelation function (1) is proportional to the autocorrelation function of the adsorbate density fluctuations within the probed area (the adsorbed particles are assumed to be mobile on the surface) [2]. At large $\tau$ the function $R_{x}(\tau)$ takes the form [2]

$$
\left.R_{x}(\tau) \propto \frac{\tau}{\tau_{0}}\left[\exp \left(-\frac{\tau_{0}}{A} \cdot\right)_{\overline{D a}}-1\right]\right]_{k i n, \text { A.G. Naumovets }}^{2}
$$

( $R_{x}$ is proportional to the function denoted by $f_{i}(t)$ in Ref. [2]). IIere $\tau_{0}$ is the characteristic time of diffusive relaxation of the adatom density fluctuations within the circular region with the radius $r_{0}$ :

$$
\tau_{0}=r_{0}^{2} / 4 D \text {, }
$$

$D$ being the adsorbate (chemical) diffusion coefficient. As follows from Eq. (4), in the limit $\tau \gg \tau_{0}$ the function $R_{x}(\tau)$ reduces to

$$
R_{x}(\tau) \propto \frac{\tau_{0}}{\tau}=\frac{r_{0}^{2}}{4 D \tau}
$$

The system $\mathrm{O} / \mathrm{W}(110)$ was studied earlier by Chen and Gomer [14] who used the FECF method in its conventional (probe hole + collector) version. They found that the asymptotic behavior of the FECF autocorrelation function is actually consistent with the model assuming diffusive relaxation of the adsorbate density fluctuations. At small $\tau$ an exponential decrease of the autocorrelation function is observed which is attributed to transitions of oxygen adatoms between different adsorption sites on W(110) ("flip-flop processes") [14, 18, 19].

Our data (Fig. 3) also agree well with the predictions of the diffusion model. This is illustrated by Fig. 4 where $R(\tau)$ is plotted in a log-log scale. It is seen

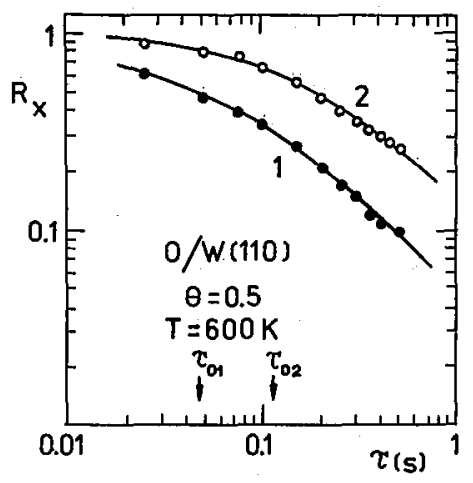

Fig. 4. A log-log plot of the data shown in Fig. 3 (points). Solid lines are curves calculated according to Gomer [2]. $\tau_{01}$ and $\tau_{02}$ (without resolution correction) are indicated by arrows.

that Gomer's theoretical curve $[2,14]$ fits experimental points quite satisfactorily. From this fitting one can extract the values of $\tau_{0}(5)$ for the two probed regions and compare the obtained ratio $\tau_{02} / \tau_{01}$ with the theoretical prediction $\tau_{02} / \tau_{01}=r_{02}^{2} / r_{01}^{2}=4$. The data of Fig. 4 give the ratio $\tau_{02} / \tau_{01}=2.4$. This 
evaluation, however, does not take into account the limited resolution of the field emission microscope. According to Gomer and Auerbach [20], a correction for limited resolution can be thade by substitution of $\tau / \tau_{0}$ in Eq. (4) by $\left(\tau / \tau_{0}\right)+2\left(\lambda / r_{0}\right)^{2}$, where $\lambda \approx 20-30 \AA$ is the resolution of the field emission microscope. Instead of Eq. (6) one has in this case $\left(\tau \gg \tau_{0}\right)$

$$
R_{x}^{-1} \propto\left[\frac{\tau}{\tau_{0}}+2\left(\frac{\lambda}{r_{0}}\right)^{2}\right] .
$$

With the resolution correction (for $\lambda=20 \AA$ ) the data of Fig. 4 give $\tau_{02} / \tau_{01}=1.8$, which differs by a factor of $\approx 2$ from the expected value.

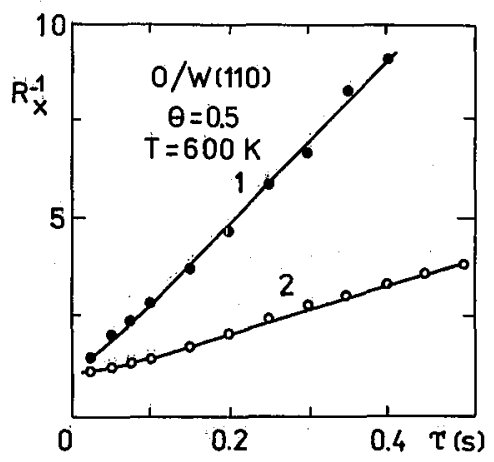

Fig. 5. Data of Fig. 3 replotted as $R_{x}^{-1}$ versus $\tau$.

In order to check the asymptotic behavior of $R_{x}$ in another way, the data of Fig. 3 were replotted as $R_{x}^{-1}$ against $\tau$ (Fig. 5). In accordance with Eqs. (6) and (7) $R_{x}^{-1}$ is linear in $\tau$ at large $\tau$.

Finally, using Eq. (6) and the absolute values $\tau_{01}$ and $\tau_{02}$ indicated in Fig. 4 , one can estimate the oxygen diffusion coefficient on $\mathrm{W}(110)$ at $T=600 \mathrm{~K}$ and $\Theta=0.5$. The average value $D \approx 2 \times 10^{-12} \mathrm{~cm}^{2} \mathrm{~s}^{-1}$ calculated from curves 1 and 2 agrees very well with the data obtained by Chen and Gomer (cf. Fig. 10 in their work [14]).

Thus, the study of the $O / W(110)$ system with the fiber-optical version of the FECF method gave results which are in general consistent with the predictions of Gomer's diffusion model [2] and with earlier results [14].

\subsection{Iydrogen on $W(110)$}

This system was investigated in detail by DiFoggio, Gomer and Wang [15, 21] who employed field emission tubes with probe holes. Hydrogen as a diffusant attracts a special interest, since at $T<160 \mathrm{~K}$ the field emission current fluctuations were found to be independent on temperature. This observation was interpreted as a manifestation of a non-activated (tunneling) diffusion, the possibility of which is assurined owing to the small mass of the hydrogen atom [15]. 
We have carried out some fluctuation measurements with a $\mathrm{H} / \mathrm{W}(110)$ system using the fiber-optical technique. We state, first of all, that our experiments confirm ther conclusion of DiFoggio and Gomer [15] about the temperature independence of field emission current fluctuations in the H/W(110) system below $160 \mathrm{~K}$.

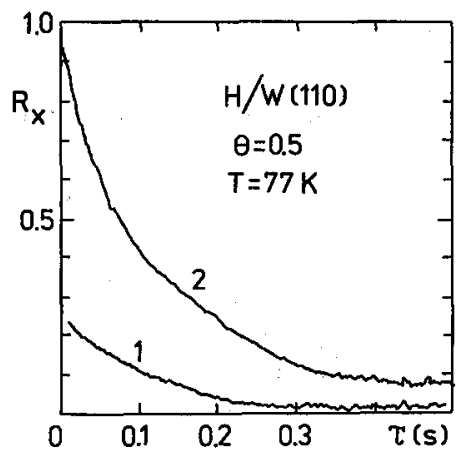

Fig. 6. Autocorrelation functions (in arbitrary units) of field emission current fluctuations for the H/W(110) system. Probed area radii are $50 \AA(1)$ and $100 \AA(2)$. Electron counting rates are $1 \times 10^{4} \mathrm{~s}^{-1}(1)$ and $2.5 \times 10^{4} \mathrm{~s}^{-1}(2)$.

Figure 6 exemplifies the autocorrelation functions measured at $T=77 \mathrm{~K}$ and a hydrogen fractional coverage of $\Theta=0.5$ for two probed regions $\left(r_{01}=50 \AA\right.$ and $r_{02}=100 \AA$ ). These data are also depicted in Fig. 7 in log-log coordinates with superimposed calculated curves. It is seen that in this case the agreement between experimental and theoretical results is less convincing than for oxygen (cf. Figs. 4 and 7 ). The $\tau_{0}$ values extracted for hydrogen are thus somewhat ambiguous.

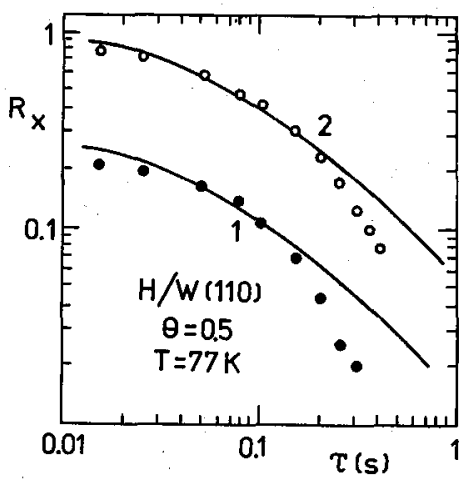

Fig. 7. A log-log plot of the data shown in Fig. 6 (points). Solid lines are curves calculated according to Gomer [2].

From the fit shown in Fig. 7 one arrives (without the resolution correction) at 
$\tau_{01}=35 \mathrm{~ms}, \tau_{02}=44 \mathrm{~ms}$ and $\tau_{02} / \tau_{01}=1.26$ instead of its expected value 4. The reason for this discrepancy remains so far unclear to us, all the more that we compared the experimental and calculated autocorrelation data in a wider range than in the work [15] (cf. e.g. our Fig. 7 with Figs. 5-7 in Ref. [15]). If, nevertheless, one uses the obtained $\tau_{01}$ and $\tau_{02}$ values to estimate the hydrogen diffusion coefficient, one obtains $D \approx 4 \times 10^{-12} \mathrm{~cm}^{2} \mathrm{~s}^{-1}$ at $T=77 \mathrm{~K}$ and $\Theta=0.5$ which is consistent with the results of DiFoggio, Gomer and Wang (see e.g. Fig. 3 in Ref. [21]). However, taking into account a somewhat uncertain agreement of the experimental and theoretical data and the existence of some other findings which are hard to reconcile with the tunneling diffusion of $H$ atoms over $W(110)[22$, 23], a further careful study of FECF mechanisms in this system still seems to be desirable.

\subsection{Silicon (111)}

The FECF method was shown to be effective in investigating not only adsorbed layers, but clean surfaces as well $[1-3,7,24,25]$. In this work we utilized the fiber-optical technique to study field emission current fluctuations for a silicon field cathode. The tip was cleaned by field evaporation. The measurements were made for two regions $\left(r_{01}=50 \AA\right.$ and $\left.r_{02}=100 \AA\right)$ within the Si(111) plane. The

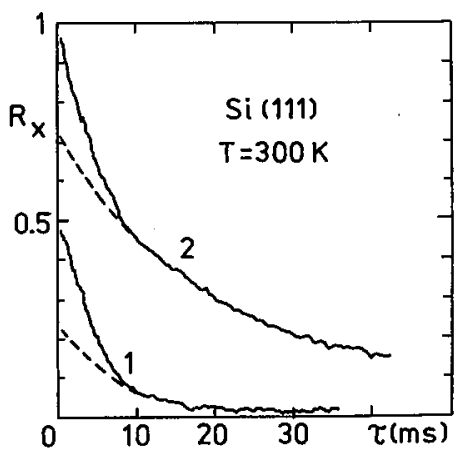

Fig. 8. Autocorrelation functions (in arbitrary units) of field emission current fluctuations for the Si(111) plane. Probed area radii are $50 \AA$ (1) and $100 \AA$ (2). Electron counting rates are $2.5 \times 10^{4} \mathrm{~s}^{-1}$ (1) and $5 \times 10^{4} \mathrm{~s}^{-1}$ (2). Extrapolations of the slowly decaying parts of the functions are shown by dashed lines.

autocorrelation functions are given in Fig. 8. They exhibit a fast (exponential) decrease at short delay times (high-frequency fluctuations) and a slower (power law) decay at long times. Similar functions were observed earlier for tungsten field emitters (see e.g. Ref. [24]).

The fast-falling section of the $R_{x}$ vs. $\tau$ curves is most naturally to be attributed to the flip-flop processes $[2,5]$ (see Sec. 3.1.). The slowly descending part is ascribed to silicon self-diffusion processes. This interpretation is consistent with the fact that upon decreasing the probed area the duration of the "fast" section 
of the $R_{x}$ vs. $\tau$ curves remains the same whereas the "slow" section shortens. R. Gomer's curve [2] calculated for the diffusion model of the field emission current fluctuations fits the autocorrelation function for Si (Fig. 8) quite well. In particular this fit gives $\tau_{02}=8 \mathrm{~ms}$ for curve 2 in Fig. 8 which results in $D \approx 3 \times 10^{-11}$ $\mathrm{cm}^{2} \mathrm{~s}^{-1}$ at $300 \mathrm{~K}$. From the temperature dependence of $\tau_{02}$ we estimated the activation energy of Si self-diffusion to be $E_{\mathrm{d}} \approx 0.35 \mathrm{eV}$ which is unexpectedly low compared to other authors' data. These range from 1 to $4 \mathrm{eV}$ and are gained from kinetical studies of such processes as surface phase transitions on stepped planes [26, 27], smoothing of rough Si surfaces observed by light scattering [28] as well as building-up and blunting of Si tips [29]. It should be noted that in all these experiments a considerable mass transport occurs which definitely involves not only the silicon "adatom" migration over smooth terraces of low-index crystal planes, but also detachment of Si atoms from steps. Perhaps the low value of the activation energy found in this work is relevant just to surface self-diffusion on smooth terraces which is not measured in more "macroscopic" methods.

\subsection{Sodium on $\mathrm{Si}$}

In contrast to the systems concidered above, the $\mathrm{Na} / \mathrm{Si}$ system is characterized by a large dipole moment of the adsorption bond. The electric field existing

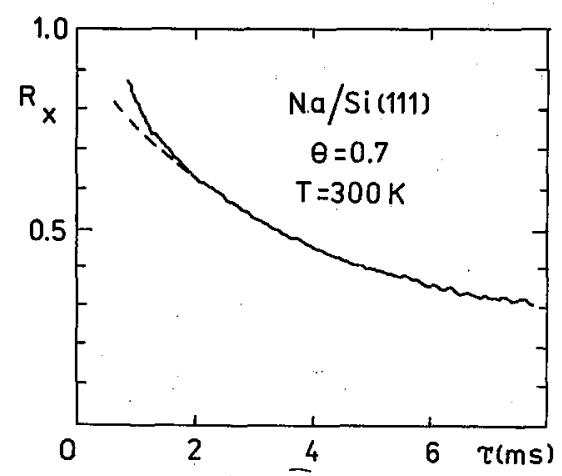

Fig. 9. Autocorrelation function (in arbitrary units) of field emission current fluctuations for the $\mathrm{Na} / \mathrm{Si}(111)$ system. $r_{0}=50 \AA$. The electron counting rate is $8 \times 10^{4} \mathrm{~s}^{-1}$.

near the tip in the FECF method can influence the kinetics of surface processes in systems with polar bond $[2,23]$. Nevertheless the application of the FECF method to such overlayers has given a lot of valuable information (see e.g. Refs. [5, 6, 8-13, $30,31])$. Moreover, field effects in surface kinetics are interesting per se.

For this reason we decided to apply the fiber-optical technique to study sodium on silicon as a representative of systems with polar adsorption bond. The autocorrelation function measured at $T=300 \mathrm{~K}$ from an area with $r_{0}=50 \AA$ on a $\mathrm{Si}(111)$ plane covered with $\mathrm{Na}(\Theta=0.7)$ is shown in Fig. 9. The diffusion coefficient $D$ estimated from Fig. $9\left(\tau_{0} \approx 1 \mathrm{~ms}\right)$ amounts to $6 \times 10^{11} \mathrm{~cm}^{2} \mathrm{~s}^{-1}$. 
On the other hand, $D$ was determined with a "shadow" technique in which one follows the spreading of the adlayer by observing field emission patterns. The tip radius was found by electron microscopy. The shadow technique gave $D=$ $1 \times 10^{-10} \mathrm{~cm}^{2} \mathrm{~s}^{-1}$. This estimation does not pretend to be of high accuracy, first of all due to the averaging character of the measurements (in the diffusion process adatoms migrate over a mosaic surface, and the coverage is also changing continuously). With this in mind we may probably state that the two $D$ values quoted above agree satisfactorily. We remind that one of them is obtained by analyzing relaxation of equilibrium adatom density fluctuations and the other by evaluation of the kinetics of an irreversible diffusion process. The fluctuation method is especially convenient in the case when the diffusion is too fast to be followed in sufficient detail by observing a transition from the non-equilibrium to the equilibrium state of the adlayer.

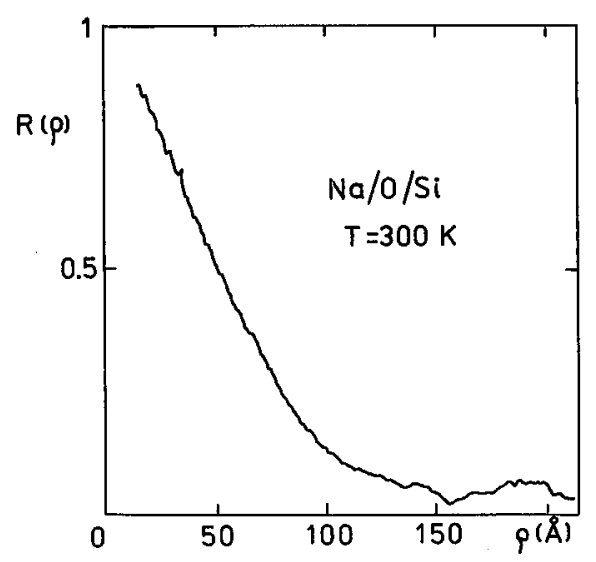

Fig. 10. Space correlation function (in arbitrary units) measured for a line of a recorded field emission pattern of $\mathrm{Na} / \mathrm{O} / \mathrm{Si}$.

We have checked also the possibility of the determination of the space correlation function (3) with the fiber-optical technique. For this purpose one can perform a correlation analysis of a line in the video-record of the field emission pattern. In our apparatus one line was recorded in $64 \mu \mathrm{s}$. This "exposure time" limits the maximum rate of the processes the influence of which can be felt on the adsorbate distribution. Figure 10 shows as an example a space correlation function $R(\rho)$ of the field emission current from a Si tip whose surface was first cleaned by field evaporation, then covered with an oxygen overlayer in the presence of an electric field and, finally, covered with a sodium monolayer. Such a procedure was employed to produce a model surface with low adsorbate mobility at room temperature. The space correlation function for this surface can be approximated as $R(\rho) \propto \exp \left(-\rho / \rho_{0}\right)$, where $\rho \approx 70 \AA$ is the average size of the work function non-uniformities in the overlayer. It was found that the correlation measurements allow to reveal a "patchy" character of the overlayer even in the case when due 
to the limited resolution of the field emission microscope the pattern has a low contrast and visually appears almost structureless.

Finally, some attempts were made to measure the cross-correlation function of FECF. We did not succeed in finding a cross-correlation in the fluctuations for any of the systems studied in this work. Distances between the probed regions down to the microscope resolution were checked. This evidences that collective fluctuation processes in the investigated systems have a rather limited spatial coherance. Of course it would be very desirable to continue experiments in this direction, since the cross-correlation measurements with electropositive adlayers on metals have revealed very interesting collective phenomena [8-13].

\section{Conclusion}

The fiber-optical version of the field emission fluctuation method presented in this work offers some essential advantages which make this method more convenient and flexible in elucidating fluctuation mechanisms and surface kinetics.

\section{Acknowledgements}

The authors would like to thank Professor P.G. Borziak for his support of this work and Professor R. Gomer for helpful discussions and sending a preprint of his review [7].

\section{References}

[1] Ch. Kleint, Surf. Sci. 25, 394 and 411 (1971).

[2] R. Gomer, Surf. Sci. 38, 373 (1973).

[3] L.W. Swanson, Surf. Sci. 70, 165 (1978).

[4] G. Mazenko, J.R. Banavar, R. Gomer, Surf. Sci. 107, 459 (1981).

[5] Ch. Kleint, Solid State Phenomena 12, 1 (1990).

[6] R. Męclewski, Solid State Phenomena 12, 49 (1990).

[7] R. Gomer, Rep. Prog. Phys. 53, 917 (1990).

[8] A. Dąbrowski, Ch. Kleint, Surf. Sci. 119, 118 (1982).

[9] R. Błaszczyszyn, Ch. Kleint, Surf. Sci. 171, 615 (1986).

[10] J. Bęben, Ch. Kleint, R. Męclewski, Surf. Sci. 213, 438 (1989).

[11] J. Bęben, Ch. Kleint, A. Pawełek, Surf. Sci. 213, 451 (1989).

[12] J.. Bęben, Solid State Phenomena 12, 17 (1990).

[13] A. Dąbrowski, Solid State Phenomena 12, 39 (1990).

[14] J.-R. Chen, R. Gomer, Surf. Sci. 79, 413 (1979).

[15] R. DiFoggio, R. Gomer, Phys. Rev. B 25, 3490 (1982).

[16] D.R. Bowman, R. Gomer, K. Muttalib, M. Tringides, Surf. Sci. 138, 581 (1984).

[17] T. Okano, T. Honda, Y. Tuzi, Jpn. J. Appl. Phys. 24, L764 (1985).

[18] M. Tringides, R. Gomer, Surf. Sci. 145, 121 (1984). 
[19] M. Tringides, R. Gomer, J. Chem. Phys. 84, 4049 (1986).

[20] R. Gomer, A. Auerbach, Surf. Sci. 167, 493 (1986).

[21] S.C. Wang, R. Gomer, J. Chem. Phys. 83, 4193 (1985).

[22] V.V. Gonchar, Y.M. Kagan, O.V. Kanash, A.G. Naumovets, A.G. Fedorus, Sov. Phys. JETP 57, 141 (1983).

[23] A.G. Naumovets, Yu.S. Vedula, Surf. Sci. Rep. 4, 365 (1984).

[24] Y.M. Gong, R. Gomer, J. Chem. Phys. 88, 1359 and 1370 (1988).

[25] D.-S. Choi, R. Gomer, Surf. Sci. 230, 277 (1990).

[26] B.Z. Olshanetsky, S.M. Repinsky, A.A. Shklyaev, Surf. Sci. 64, 224 (1977).

[27] B.Z. Olshanetsky, A.A. Shklyaev, Zh. Eksp. Teor. Fiz. 81, 361 (1981) (in Russian; English translation in Sov. Phys. JETP).

[28] Yu.L. Gavrilyuk, Yu.S. Kaganovsky, V.G. Lifshits, K'ristallografiya 26, 561 (1981) (in Russian; English translation in Sov. Phys.-Crystallogr.).

[29] P.G. Borziak, A.A. Dadykin, Izv. Akad. Nauk SSSR Ser. Fiz. (USSR) 46, 1288 (1982) (in Russian; English translation in Bull. USSR Acad. Sci., Phys. Ser.).

[30] R. Morin, Surf. Sci. 155, 187 (1985).

[31] R. Morin, Surf. Sci. 162, 109 (1985). 